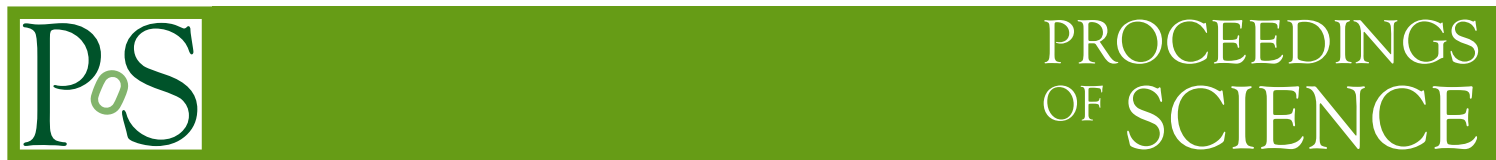

\title{
Observation of single top at CDF
}

\section{Bruno Casal* (on behalf of the CDF collaboration)}

Instituto de Física de Cantabria (CSIC - Univ. Cantabria). - Avda. Castros s/n, 39005

Santander, Spain

E-mail: Casalefnal.gov

We present observation of electroweak single top quark production using $3.2 \mathrm{fb}^{-} 1$ of data collected by the CDF experiment. Candidate events are selected for further classification by five parallel analysis techniques: one using a likelihood discriminant, one using a matrix-element discriminant, one using decision trees, one using a neural network, and one using a complementary dataset. The results of these analyses are combined in order to improve the expected sensitivity. The signicance of the observed data is 5.0 standard deviations, and the expected sensitivity is in excess of 5.9 standard deviations. We also present the most current value of the CKM matrix element $\left|V_{t b}\right|$.

European Physical Society Europhysics Conference on High Energy Physics, EPS-HEP 2009, July 16 - 222009

Krakow, Poland

\footnotetext{
* Speaker.
} 


\section{Introduction}

The establishment of the presence of the electroweak production of single top quarks in $p \bar{p}$ collisions is an important goal of the Tevatron program. The reasons for studying single top quarks are compelling: the production cross section is directly proportional to the square of the CKM matrix element $\left|V_{t b}\right|$ [1], and thus a measurement of the rate constrains fourth-generation models, models with flavor-changing neutral currents, and other new phenomena [2]. Furthermore, understanding single top quark production provides a solid anchor to test the analysis techniques that are also used to search for Higgs boson production and other more speculative phenomena.

In the SM, top quarks are expected to be produced singly through $t$-channel or $s$-channel exchange of a virtual $W$ boson. This electroweak production of single top quarks is a really difficult process to measure because the expected combined production cross section $\left(\sigma_{s+t} \sim 2.9 \mathrm{pb}\right.$ [3, 4] $)$ is much smaller than those of competing background processes. Also, the presence of only one top quark in the event provides fewer features to use in separating the signal from background, compared with measurements of top pair production $(t \bar{t})$, which was first observed in 1995 [5].

Both the CDF and D0 collaborations at the Tevatron have published evidence for single top quark production at significance levels of 3.7 and 3.6 standard deviations, respectively [6, 7]. More recently both collaborations have reported observation of single top quark production [8, 9], this document describes the latest analyses done using $3.2 \mathrm{fb}^{-1}$ of data collected with the CDF II [10] detector leading to the observation of single top quark production.

\section{Event Selection and Backgrounds}

For the analyses shown here, we assume that single top quarks are produced in the $s$ - and $t$-channel modes with the SM ratio, and that the branching fraction of the top quark to $\mathrm{Wb}$ is $100 \%$ (corresponding to $\left|V_{t b}\right|>>\left|V_{t s}\right|,\left|V_{t d}\right|$ ). For most of the analysis channels, we seek events in which the $W$ boson decays leptonically in order to improve the signal-to-background ratio.

The basic event selection is based on selecting $\ell+E_{T}+$ jets events, where $\ell$ is an explicitly reconstructed electron or muon from the $W$ boson decay. This lepton is required to be isolated from nearby jets and to have large transverse momentum, $p_{T}>20 \mathrm{GeV}$. The presence of high missing transverse energy, $E_{T}>25 \mathrm{GeV}$, and two or three energetic jets, $E_{T}>20 \mathrm{GeV}$, are also required. At least one of the jets has to be identified as a jet coming from a $b$-quark.

The background has contributions from events in which a $W$ boson is produced in association with one or more heavy flavor jets, events with mistakenly $b$-tagged light-flavor jets, multijet events (QCD), $t \bar{t}$ production, diboson processes, and $Z+$ jet events.

The expected number of $\ell+H_{T}+$ jets events as a function of the number of jets for the signal and each background process is shown in Fig. 1(a). The two and the three jet bins are used for the search of the single top signal. From this figure, it is clear that the signal is hidden under huge and uncertain backgorunds which make counting experiments impossible.

\section{Multivariate Analysis}

To overcome these challenges, a variety of multivariate techniques for separating single top events from the backgrounds have been developed as described below. 


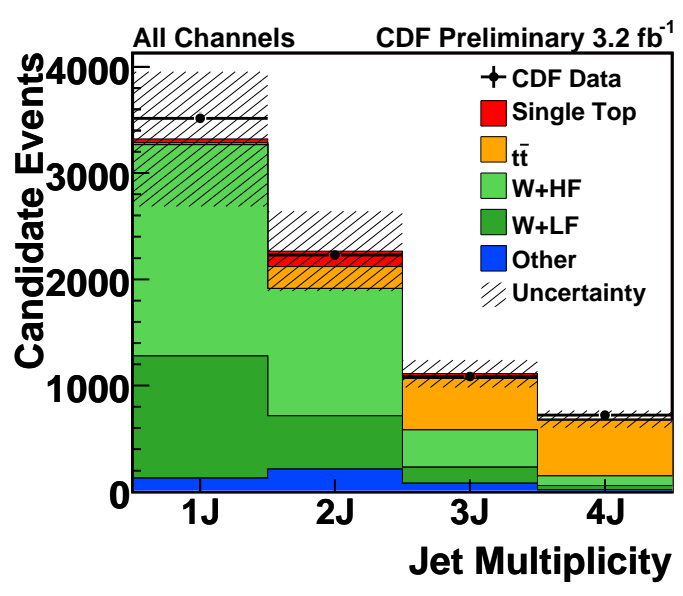

(a)

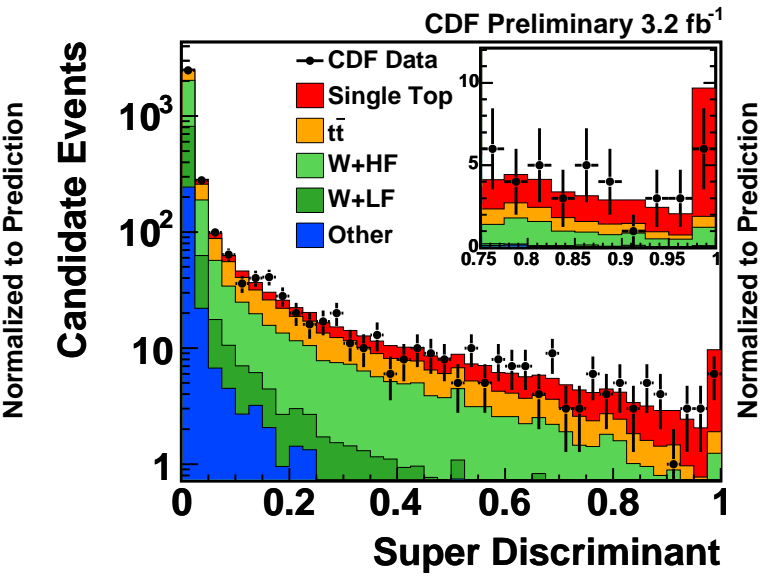

(b)

Figure 1: (a) Expected number of $\ell+E_{T}+$ jets events as a function of the number of jets for the signal (red) and each background process; the dashed band is the uncertainty in the background predictions. (b) CDF discriminant output distribution of the combination of the $\ell+E_{T}+$ jets analyses.

\subsection{Likelihood Function (LF)}

A projective likelihood technique [11, 12] is used to combine information from several input variables to optimize the separation of the single top signal from the backgrounds. Two likelihood functions are created, one for two jet events, and one for three jet events with 7 and 10 input variables, respectively. Some of the input variables used are: the total scalar sum of transverse energy in the event $H_{T}, Q \times \eta[14]$, the dijet mass $M_{j j}, \cos \theta_{l j}^{*}[15]$ and the t-channel matrix element.

A new separate analysis dedicated to the search for single top quark production in the s-channel is also performed using this technique (LFS) [13]. In this case, the likelihood function is optimized to be sensitive to the $s$-channel process using the subset of the $\ell+E_{T}+$ jets sample with two $b$-tagged jets.

\subsection{Neural Networks (NN)}

This approach [16] employs neural networks which combine many variables into one more powerfull discriminant and have the general advantage that correlations between the discriminating input variables are identified and utilized to optimize the separation power between signal and background.

Four separate networks are trained to identify different signals in distinct samples using simulated events from the common samples described previously. The networks use 11 to 18 input variables. Among the most important ones are $Q \times \eta, M_{j j}, \cos \theta_{l j}^{*}$, the invariant mass of the $\ell v b$ system $M_{\ell v b}$, and the output of a jet-flavor separator dedicated neural network [17].

\subsection{Matrix Elements (ME)}

The ME method [18] relies on the evaluation of event probability densities for signal and background processes based on calculations of the standard model differential cross sections [19]. 
We construct these probability densities for each signal and background process for each event given their measured quantities $x$ by integrating the appropriate differential cross section $d \sigma(y) / d y$ over the underlying partonic quantities $y$, convolved with the parton distribution functions (PDFs) and detector resolution effects.

The event probability densities are combined into an event probability discriminant: $E P D=$ $P_{\text {signal }} /\left(P_{\text {signal }}+P_{\text {background }}\right)$. To better classify signal events that contain $b$ jets, the output of the neural network jet-flavor separator is incorporated into the final discriminant.

\subsection{Boosted Decission Trees (BDT)}

The BDT discriminant uses a decision tree method that applies binary cuts iteratively to classify events [21]. The discrimination is further improved using a boosting algorithm [22]. The BDT discriminant uses over 20 input variables, some of the most sensitive are the neural network jet-flavor separator, $M_{\ell v b}, H_{T}, Q \times \eta, M_{j j}$, and the transverse mass of the $W$ boson.

\section{5 $B_{T}+$ jets $(\mathbf{M J})$}

The MJ analysis is a new analysis in CDF, using data corresponding to $2.1 \mathrm{fb}^{-1}$ of integrated luminosity, designed to select events with $H_{T}$ and jets, while vetoing events selected by the $\ell+$ $E_{T}+$ jet analyses. It accepts events in which the $W$ boson decays into $\tau$ leptons and those in which the electron or muon fails the lepton identification criteria.

The advantage of this analysis is that it is orthogonal to the $\ell+E_{T}+$ jets analysis described above, increasing the signal acceptance by $\sim 30 \%$. The disadvantage is the huge instrumental background due to QCD events in which mismeasured jet energies produce large $\vec{B}_{T}$ aligned in the same direction as jets. To reduce this background, a QCD removal neural network is used suppressing 77\% of the QCD background while keeping 91\% of the signal acceptance.

Finally, the MJ discriminant uses a neural network to combine information from several input variables. The most important variables are the invariant mass of the $\vec{B}_{T}$ and the second leading jet, the scalar sum of the jet energies, the $\mathbb{E}_{T}$, and the azimuthal angle between the $\vec{E}_{T}$ and the jets.

\subsection{Combination}

CDF combines the LF, ME, NN, BDT, and LFS channels using a super-discriminant (SD) technique. The SD method uses a neural network trained with neuro-evolution [23] to separate the signal from the background taking as inputs the discriminant outputs of the five analyses for each event. A simultaneous fit over the two exclusive channels, MJ and SD, is performed to obtain the final combined results. For illustrative purposes, Fig. 1(b) shows the distributions of the $\ell+E_{T}+$ jets super discriminant combination.

\section{Statistical Methods}

The cross section is measured using a Bayesian binned likelihood technique [20] assuming a flat non-negative prior in the cross section and integrating over the systematic uncertainties. The measured cross section is quoted as the position of the peak of the posterior density distribution and the shortest interval containing $68 \%$ of the integral of the posterior is used to set the \pm 1 sigma uncertainties. 
The significance is calculated as a $p$-value [20], which is the probability, assuming the absence of single top quark production, that the value of the test statistic, $-2 \ln Q=-2 \ln \left(\frac{p(\text { data } \mid s+b)}{p(\text { data } \mid b)}\right)$, is smaller (or more signal like) than that observed in the data. The $p$-value is then converted into a number of standard deviations using the integral of one side of a Gaussian function.

\section{Systematics}

All sources of systematic uncertainty are included and correlations between normalization and discriminant shape changes are considered. Uncertainties in the jet energy scale, $b$-tagging efficiencies, background modeling, lepton identification and trigger efficiencies, the amount of initial and final state radiation, PDFs, and factorization and renormalization scale have been explored and incorporated in all individual analyses and the combination.

\section{Results}

Table 1 lists the cross sections and the observed and expected significances for each of the component analyses and the combination of them. The excess of signal-like events over the expected background is interpreted as observation of single top production with a $p$-value of about $3.10 \times 10^{-7}$, corresponding in both cases to a signal significance of 5.0 standard deviations. The sensitivity is defined to be the median expected significance and is found to be in excess of 5.9 standard deviations.

CDF finds a value of the combined $s$-channel and $t$-channel cross sections of $2.3_{-0.5}^{+0.6} \mathrm{pb}$ assuming a top quark mass of $175 \mathrm{GeV} / c 2$.

Since the CKM matrix element $\left|V_{t b}\right|^{2}$ is proportional to the cross section, its value can be directly measured. From the cross section measurement at $m_{t}=175 \mathrm{GeV} / c 2$, CDF obtains $\left|V_{t b}\right|=$ $0.91 \pm 0.11$ (stat + syst) \pm 0.07 (theory [[] ) and a limit $\left|V_{t b}\right|>0.71$ at the $95 \%$ C.L. A flat prior in $\left|V_{t b}\right|^{2}$ from 0 to 1 is assumed for the $95 \%$ CL limit results.

\begin{tabular}{c|ccccc|cc|c}
\hline \hline Analysis & LF & ME & NN & BDT & LFS & SD & MJ & Comb. \\
\hline Cross Section $(\mathrm{pb})$ & $1.6_{-0.7}^{+0.8}$ & $2.5_{-0.6}^{+0.7}$ & $1.8_{-0.6}^{+0.6}$ & $2.1_{-0.6}^{+0.7}$ & $1.5_{-0.8}^{+0.9}$ & $2.1_{-0.5}^{+0.6}$ & $4.9_{-2.2}^{+2.5}$ & $2.3_{-0.5}^{+0.6}$ \\
Obs. Sign. $(\sigma)$ & 2.4 & 4.3 & 3.5 & 3.5 & 2.0 & 4.8 & 2.1 & 5.0 \\
Exp. Sign. $(\sigma)$ & 4.0 & 4.9 & 5.2 & 5.2 & 1.1 & $>5.9$ & 1.4 & $>5.9$ \\
\hline \hline
\end{tabular}

Table 1: Results summary for the five correlated $\ell+E_{T}+$ jets analyses combined by the SD analysis, the SD and the MJ analysis, and the total combination. The LFS analysis measures only the $s$-channel production cross section, while the other analyses measure the sum of the $s$ - and $t$-channel cross sections.

\section{Conclusions}

In summary, the CDF collaboration has developed several multivariate analysis techniques to distinguish single top signal from background events and has combined them to precisely measure the electroweak single top production cross section and the CKM matrix element $\left|V_{t b}\right|$. Single top 
production has been observed with a significance of 5.0 standard deviations. More details can be found in the CDF public web page [24].

\section{References}

[1] N. Cabibbo, Phys. Rev. Lett. 10, 531 (1963);

M. Kobayashi and T. Maskawa, Prog. Theor. Phys. 49, 652 (1973).

[2] T. M. P. Tait and C. P. Yuan, Phys. Rev. D 63, 014018 (2001).

[3] B. W. Harris et al., Phys. Rev. D 66, 054024 (2002).

[4] Z. Sullivan, Phys. Rev. D 70, 114012 (2004);

J. Campbell, K. Ellis, and F. Tramontano, ibid. 70, 094012 (2004);

N. Kidonakis, ibid. 74, 114012 (2006).

[5] F. Abe et al. (CDF Collaboration), Phys. Rev. Lett. 74, 2626 (1995);

S. Abachi et al. (D0 Collaboration), ibid. 74, 2632 (1995).

[6] T. Aaltonen et al. (CDF Collaboration), Phys. Rev. Lett. 101, 252001 (2008).

[7] V. M. Abazov et al. (D0 Collaboration), Phys. Rev. Lett. 98, 181802 (2007);

V. M. Abazov et al. (D0 Collaboration), Phys. Rev. D 78, 012005 (2008).

[8] T. Aaltonen et al. (CDF Collaboration), Phys. Rev. Lett. 103, 092002 (2009).

[9] V. M. Abazov et al. (D0 Collaboration), Phys. Rev. Lett. 103, 092001 (2009).

[10] D. Acosta et al. (CDF Collaboration), Phys. Rev. D 71, 032001 (2005).

[11] K. Ackerstaff et al. (OPAL Collaboration), Eur. Phys. J. C 1, 425 (1998).

[12] S. Budd, Ph.D. thesis, Illinois University, Urbana, FERMILAB-THESIS-2008-30 (2008).

[13] K. Nakamura, Ph.D. thesis, Tsukuba University, FERMILAB-THESIS-2009-13 (2009).

[14] $Q \times \eta$ is the charge of the lepton times the pseudorapidity of the jet not assigned to be the $b$ from top quark decay; see C. P. Yuan, Phys. Rev. D 41, 42 (1990).

[15] $\cos \theta_{l j}^{*}$ is the cosine of the angle between the lepton and the jet not assigned to be the $b$ in the top decay, in the top quark rest frame. G. Mahlon and S. J. Parke, Phys. Rev. D 55, 7249 (1997).

[16] J. Lueck, Ph.D. thesis, University of Karlsruhe, FERMILAB-THESIS-2009-33 (2009).

[17] S. Richter, Ph.D. thesis, University of Karlsruhe, FERMILAB-THESIS-2007-35 (2007).

[18] P. Dong, Ph.D. thesis, University of California, Los Angeles, FERMILAB-THESIS-2008-12 (2008).

[19] V. M. Abazov et al. (D0 Collaboration), Nature 429, 638 (2004).

[20] C. Amsler et al. (Particle Data Group), Phys. Lett. B 667, 1 (2008).

[21] L. Breiman et al., Classification and Regression Trees, (Wadsworth and Brooks, Monterey, CA, 1984).

[22] A. Höcker et al., PoS A CAT, 040 (2007) [arXiv:physics/0703039].

[23] K. O. Stanley and R. Miikkulainen, Evolutionary Computation 10 (2) 99-127 (2002). S. Whiteson and D. Whiteson, arXiv:hep-ex/0607012 (2006).

[24] CDF public web page: http://www-cdf.fnal.gov/physics/new/top/public_singletop.html. 